\title{
Selective photo-dissociative ionization of methane molecule with TDDFT study
}

\author{
E. Irani, A. Anvari ${ }^{*}$ and R. Sadighi- Bonabi ${ }^{\dagger}$ \\ Department of Physics, Sharif University of Technology, P.O. Box 11365-9567, Tehran, Iran
}

\begin{abstract}
Three dimensional calculation of control dynamics for finding the optimized laser filed is formulated using an iterative method and time-dependent density functional approach. An appropriate laser pulse is designed to control the desired products in the dissociation of methane molecule. The tailored laser pulse profile, eigenstate distributions and evolution of the efficient occupation numbers are predicted and exact energy levels of this five-atomic molecule is obtained. Dissociation rates of up to $78 \%, 80 \%, 90 \%$, and $82 \%$ for $\mathrm{CH}_{2}{ }^{+}, \mathrm{CH}^{+}, \mathrm{C}^{+}$and $\mathrm{C}^{++}$are achieved. Based on the present approach one can reduce the controlling costs.
\end{abstract}

Keywords: methane molecule, femtosecond laser, dissociative ionization, optimal control theory.

\section{INTRODUCTION}

One of the attractive methods in the dissociation of molecules is using intense pulse shaped lasers. Interaction of strong laser fields with molecules leads to nonlinear effects, including multiphoton ionization, above-threshold ionization, tunneling and rescattering ionization, Coulomb explosion, charge resonance enhanced ionization, and field assisted dissociation and high harmonic generation [1-5]. Recent progress of control of electron wave packets, probing of nuclear and electronic dynamics, imaging of molecular orbitals with ultra-fast laser pulses have key roles for coherent control of photochemical reactions [6-8]. The capability of controlling the dynamic of molecules by ultra-short laser pulse in order to transfer an initial state to a desired thermally inaccessible state was suggested by Tannor et al. in 1985 [9]. The visualization and

\footnotetext{
Email address: *anvari@ sharif.ir

Email address: ${ }^{\dagger}$ Sadighi@sharif.ir
} 
analysis of the dynamics of chemical bonds shortly implemented by real time observations of the transition-state region between reactants and products in 1994 [10]. The pulse shaping technology had developed over the last decades, and the experimental optimal control had translated from theoretical methods to a unique experiment by Judson and Rabitz [11]. For more complex systems and clusters, a feedback loop, together with a learning algorithm are used to achieve the preferred goal [12].

Quantum optimal control theory (QOCT) provides the necessary tools to the tailored theoretical design of the laser pulses for controlling a molecular system towards preferred reaction pathways [13]. For strong field ionization and dissociation processes, there have been many theoretical studies which can be considered as possible calculations of the strong field approximations which result in few simple theoretical expressions [14-15]. However, at the mentioned high powerful lasers, these models fail to explain some experimental observations when multielectron roles, inner orbital effects and ion-electron interactions are considerable [16]. Due to these inherent limitations exact numerical solutions of the strong field dynamics for the large molecules did not exist and a complete theoretical study for controlling dynamics is still attractive and desirable. Recently, only global mathematical procedure is suggested to use OCT with time-dependent density functional theory (TDDFT) for a quantum system by Castro et al. [17]. To the best of our knowledge, no theoretical calculations for selective quantum optimal control of desired fragments are reported beyond the two atomic molecules due to their complexity of orbitals, inner electron effects and the numerous interaction forces among them.

The present work, concentrates on the dissociation of methane molecule, one of the major greenhouse gases, and the control of reactions into the valuable fragments. The valuable fragmental ions of $\mathrm{CH}_{2}{ }^{+}, \mathrm{CH}^{+}, \mathrm{C}^{+}$and $\mathrm{C}^{++}$created from $10^{14}-10^{15} \mathrm{Wcm}^{-2}$ intensity range are 
formed by Coulomb explosion. Due to the multi-orbital effects, molecular dynamics analysis and controlling of molecular dynamics is considerably complicated. In this paper, following our recent theoretical and experimental studies on the dissociation of methane [18-20], the optimized laser field via convolution strategy of TDDFT with QOCT is obtained. The focus is to improve the dissociation rate and selective control of the dissociation path by considering the modified pulse shapes to facilitate several possible transitions in a multi-atomic molecule. This approach extracts the exact dynamics of the excited electrons and time evolution of the most important occupation numbers by suitably tailored laser pulse and also an exact energy diagram can be presented. Based on the obtained energy diagram, dissociation pathways are clearly identified for valuable ionic fragmentations of $\mathrm{CH}_{2}{ }^{+}, \mathrm{CH}^{+}, \mathrm{C}^{+}$and $\mathrm{C}^{++}$. Indeed, the great improvements of dissociation rates are achieved in controlling product ratios of desired products with theoretically designed laser field. The simulations indicate that direct closed loop control in the laboratory operates effectively with less complexity and low cost method.

This paper is organized as follows. In Sec. II, time -dependent density functional approach and quantum optimal control scheme are introduced to provide tailored femtosecond laser pulses for an enhanced dissociation rates and capable of controlling a system towards the prescribed products. In Sec. III, the calculated results are explained in detail. The paper is concluded in Sec. IV.

\section{Computational method}

\section{A. Time -Dependent Density Functional Approach}

In this calculation, a numerical grid in the three dimensional real space are used as $\Delta \mathrm{r}=0.01$ a.u. and $\Delta \mathrm{t}=0.02 \mathrm{a}$.u. This is controlled by the variable radius of sphere box shape [21-24]. The radius of spherical simulation box is $30 \mathrm{a} . \mathrm{u}$. and the box is surrounded by absorbing boundaries of 
a $\sin ^{2}$-type imaginary absorber at the edges of grid which smoothly brings down the wave function without spurious reflections. In order to study spatial asymptotic effects on the exchange-correlation (XC) potential, the time-dependent Kohn-Sham equations are numerically solved with different $\mathrm{XC}$ potentials. The $\mathrm{XC}$ potential with $\mathrm{TD}-\mathrm{LB}_{\alpha}$ potential is more accurate to investigate the electronic structure of methane and dissociation yields [25].

The electron density $\rho(\mathbf{r}, \mathrm{t})$ at the electron coordinate $\mathbf{r}$ and time $\mathrm{t}$ is determined by

$\rho(\mathbf{r}, t)=\sum_{\sigma} \sum_{i=1}^{N \sigma} \psi_{i \sigma}^{*}(\mathbf{r}, t) \psi_{i \sigma}(\mathbf{r}, t)$

$\sigma$, $\mathrm{i}$ and $\mathrm{N}_{\sigma}$ are spin index, orbital index and the number of electrons with $\sigma$ spin, respectively. $\Psi_{\text {i } \sigma}(\mathbf{r}, \mathrm{t})$ satisfies the time dependent Kohn-sham equation, in atomic units. The Kohn-Sham potential is conventionally separated in the following way

$i \frac{\partial}{\partial t} \psi_{i \sigma}(\mathbf{r}, t)=\left\{-\frac{\nabla^{2}}{2}+V_{K s h}(\mathbf{r}, t)\right\} \psi_{i \sigma}(\mathbf{r}, t)=\left\{-\frac{1}{2} \nabla^{2}+V_{n e}(\mathbf{r})+V_{H}(\mathbf{r}, t)+V_{x c}(\mathbf{r}, t)+V_{e x t}(\mathbf{r}, t)\right\} \psi_{i \sigma}(\mathbf{r}, t)$,

$\mathrm{V}_{\text {ne }}(\mathbf{r})$ is the potential of electron interaction with the nuclei, $\mathrm{V}_{\mathrm{H}}(\mathbf{r}, \mathrm{t})$ is Hartree potential, $\mathrm{V}_{\mathrm{xc}}(\mathbf{r}$, t) is $\mathrm{TD}-\mathrm{LB}_{\alpha}$ (van Leeuwen-Baerends) exchange-correlation potential. $\mathrm{V}_{\text {ext }}(\mathbf{r}, \mathrm{t})$ is the external potential which describes the interaction of molecule with the laser field and it is expressed rely on dipole approximation as follows

$V_{e x t}(\mathbf{r}, t)=f(t) \mathbf{E}_{\mathbf{0}} \sin (\omega t) \cdot \mathbf{r}$

where $\mathbf{E}_{\mathbf{0}}, \mathrm{f}(\mathrm{t})$ and $\omega$ are the peak field strength in the $\mathrm{Z}$ direction, the temporal profile and the carrier frequency, respectively.

The propagation of wave function is implemented by second order split-operator scheme

$\psi_{i \sigma}\left(\mathbf{r}, t_{0}+\Delta t\right)=\exp \left(-i \hat{V}_{K s h}(\mathbf{r}, t) \Delta t / 2\right) F^{-1}\left\{\exp (-i \hat{T} \Delta t) F\left[\exp \left(-i \hat{V}_{K s h}(\mathbf{r}, t) \Delta t / 2\right) \psi_{i \sigma}\left(\mathbf{r}, t_{0}\right)\right]\right\}+O\left(\Delta t^{3}\right)$, 
$\hat{T}$ and $\hat{V}_{K s h}(\mathbf{r}, t)$ are kinetic energy and Kohn-Sham potential, respectively. Within the temporal duration of the laser pulse, the potential surfaces are modified by the laser field and if the laser intensity is strong enough, it can induce the ion dissociation fragments. Methane is a symmetric molecule and the ground electronic state configuration of the five doubly occupied molecular orbitals is $\left(1 a_{1}\right)^{2}\left(2 a_{1}\right)^{2}\left(1 t_{2}\right)^{6}$. Molecular excitation with these intense lasers can be interpreted as a transition from the bound state to the unbound state and breaking $\mathrm{C}-\mathrm{H}$ bond lengths as one minus the projection to 40 lowest eigenstates. The electron density that leaves 18a.u.-50a.u. box radius, during the interaction with the laser field with wavelength of $800 \mathrm{~nm}$ and intensity in the range of $10^{14}-10^{15} \mathrm{Wcm}^{-2}$ represents unbound states and molecule will be dissociated. This box radius approximately agrees with the spatial extent of 40 lowest eigenstates. The total dissociation probability is calculated from a sum over the populations of all unbound states or, alternatively, one minus the sum over all bound state populations which can be calculated by

$P_{\text {diss }}=1-\sum_{i}^{\text {bound }}\left|\left\langle\psi_{i}(\mathbf{r}) \mid \psi(\mathbf{r}, t)\right\rangle\right|^{2}$

Dissociation probability is calculated for interaction of $\mathrm{CH}_{4}$ molecule with initial intense Ti: Sapphire laser pulse with pulse duration of 20fs (FWHM), rectangular envelope and peak laser intensities in the range of $10^{14} \mathrm{Wcm}^{-2}-10^{15} \mathrm{Wcm}^{-2}$ using octopus code [26].

\section{B. Pulse Optimization with Quantum Optimal Control Theory}

QOCT is used for finding the optimized laser pulse that guides the molecular system to predefined products with the maximum efficiency. The results are promising by combining of QOCT with TDDFT in order to obtain an accurate energy diagram and indentify dissociation pathways for valuable ionic fragmentations. The shape of laser pulse is now capable to cover 
the other transitions for several possible pathways, properly. In this efficient and low cost method, without requiring experimental data, an optimum pulse shape with the maximum yield is introduced. The objective of optimal control theory can be formulated as an optimal laser pulse $\varepsilon(\mathrm{t})$ which drives the state $|\Psi(\mathbf{r}, \mathrm{t})\rangle$ of the system in a finite time interval $\mathrm{T}$ from an initial state $\left|\Phi_{\mathrm{i}}\right\rangle$ to a prescribed target state $\left|\Phi_{\mathrm{f}}\right\rangle$. The optimum condition corresponds to the maximum of following overlap functional

$$
J_{1}=|<\psi(\mathbf{r}, T)| \phi_{f}>\left.\right|^{2}
$$

To adjust the significance of the laser intensity, the total fluence of laser pulse is weighted with a penalty factor $\alpha$

$$
J_{2}=-\alpha\left[\int_{0}^{T} d t \varepsilon^{2}(t)-E_{0}\right]
$$

where $\mathrm{E}_{0}$ is the initial fluence. Finally, the system obeys the time-dependent Schrödinger equation, that the state $|\Psi(\mathbf{r}, \mathrm{t})\rangle$ actually represents a time-evolved quantum state. This can be expressed by

$J_{3}=-2 \operatorname{Im} \int_{0}^{T} d t<\kappa(r, t)\left|\left(i \partial_{t}-H(r, t)\right)\right| \psi(r, t)>$,

$\kappa(\mathbf{r}, \mathrm{t})$ is a Lagrange multiplier. The optimal laser pulse $\varepsilon(\mathrm{t})$ for the transition of initial state into favorite final state maximizes the total functional $J=J_{1}+J_{2}+J_{3}$.

The extrema of $\mathbf{J}$ is characterized by setting the total variation of the functional to zero.

$\delta_{\psi} J=0:\left(i \partial_{t}-H(\mathbf{r}, t)\right) \mid \kappa(\mathbf{r}, t)>=i\left(|\kappa(\mathbf{r}, t)>-| \phi_{f}><\phi_{f} \mid \psi(\mathbf{r}, t)>\right) \delta(t-T)$,

$\delta_{\kappa} J=0:\left(i \partial_{t}-H(\mathbf{r}, t)\right)|\psi(\mathbf{r}, t)>=0,| \psi(\mathbf{r}, 0)>=\mid \phi_{i}(\mathbf{r})$,

$\delta_{\varepsilon} J=0: \alpha \varepsilon(t)=-\operatorname{Im}<\kappa(\mathbf{r}, t)|\mu| \psi(\mathbf{r}, t)$,

For solution $|\kappa(\mathbf{r}, t)\rangle$, the first equation is equivalent to 
$\left(i \partial_{t}-H(\mathbf{r}, t)\right)|\kappa(\mathbf{r}, t)>=0,| \kappa(\mathbf{r}, t)=\mid \phi_{f}><\phi_{f} \| \psi(\mathbf{r}, T)>$

which shows that the Lagrange multiplier satisfies the time dependent Schrödinger equation with an initial condition at the end of the time interval at $\mathrm{t}=\mathrm{T}$. The obtained expressions together with the boundary conditions implement the control equations. These equations are solved with an iterative method and forward-backward scheme with octopus code [26, 27].

\section{RESULTS}

To study the dissociation scheme and possible photo-dissociation channels, geometry optimization and the energies of ions are computed using CCSD (T)/cc-pVQZ level [28]. Connections between transition states and local minima are checked by intrinsic reaction coordinate (IRC) calculations. The dissociation pathways for ionic fragmentations are illustrated in the presented energy diagram (Fig. 1).

It is assumed that ionization occurs immediately after excitation of the parent molecule by a strong laser with energy higher than the ionization threshold. From Fig. 1, a number of different product channels are feasible. The ionic fragmentations of $\mathrm{CH}_{2}^{+}, \mathrm{CH}^{+}, \mathrm{C}^{+}$and $\mathrm{C}^{++}$, are valuable products and the goal of the oil and gas industry.

Using the optimal control equations and time-dependent density functional theory, optimal laser pulses which drives the system from the ground state to the different excited states, leading to the products of $\mathrm{CH}_{2}^{+}, \mathrm{CH}^{+}, \mathrm{C}^{+}$and $\mathrm{C}^{++}$are simulated. The initial laser field before the optimization is a linearly polarized pulse having a rectangular envelope, wavelength of $800 \mathrm{~nm}$, FWHM of 20fs and peak laser intensity in the range of $10^{14}-10^{15} \mathrm{Wcm}^{-2}$ for the different ionic fragments. Calculations are carried out for penalty factor $\alpha=1.0$ for 70 iteration counts. The results of the optimization which transfers the ground state to the 3th excited state as the target functional, 
leading to the final product of $\mathrm{CH}_{2}{ }^{+}+2 \mathrm{H}+\mathrm{e}$, are presented in Fig. 2. The functional tries to find a laser field which produces a high occupation and optimal fluence. The methodology looks for pulses that maintain the same total length and integrated intensity as a given pulse that serves as an initial guess. Fig. 2(a) shows the optimized pulse in comparison with the initial pulse. Based on optimized pulse, the optimization reduces the peak intensity and increases the time duration pulse with the fluence 0.57 a.u. for $\mathrm{CH}_{2}{ }^{+}$product which this value reduces to half the reported experimental value for $5 \times 10^{14} \mathrm{Wcm}^{-2}$ laser intensity [29]. The Fourier transform of the optimized laser pulse clarifies that the tailored field has more contributions from the frequencies corresponding to the different vibrational transitions which are presented the main contribution in energy diagram of Fig.1. Applying this tailored laser field to the reaction system yields an occupation of $78 \%$ in the target state. This behavior is visible in Fig. 2(b), where the evolution of occupation numbers as a function of time is shown.

Indeed, the optimized pulse strongly couples with the (de)-excitations of the system, which leads to different pulse characteristics. Therefore, the optimal laser pulse is convolution of several transition frequencies rather than a single frequency corresponding to the energy gap between the initial and the final states. The Fourier transform of the optimized laser pulse clarifies that the tailored field has major contribution from the frequencies corresponding to the different vibrational transitions. Along the path of this transition, the optimized process leads to $78 \% \mathrm{CH}_{2}{ }^{+}$ yield with the optimal fluence. The occupation of lowest unoccupied molecular orbital (LUMO), $3 \mathrm{a}_{1}$ and three doubly degenerate LUMOs of methane, $2 \mathrm{t}_{2}$ are calculated, $4 \%, 38 \%, 30 \%$ and $6 \%$, respectively. For $5 \times 10^{14} \mathrm{Wcm}^{-2}$ and $10^{15} \mathrm{Wcm}^{-2}$ laser intensities, total dissociation yields of methane molecule are obtained about $10 \%$ and $15 \%$ [19]. The experimental fragmental ion yields 
of $\mathrm{CH}_{2}{ }^{+}, \mathrm{CH}^{+}, \mathrm{C}^{+}$and $\mathrm{C}^{2+}$ for $10^{14} \mathrm{Wcm}^{-2}-10^{15} \mathrm{Wcm}^{-2}$ intensity range and pulse duration range from $35 f$ f to 48 fs are much lower than the induced optimization results [30, 31].

Moreover, the four lowest eigenstate distributions in the plane XY are presented in Fig. 3. The above figures illustrate that the optimized laser pulse drives the wavefunctions in the desired way. By radiation of the laser field, the wave packet moves on the potential energy surfaces. In the first half of the optical cycle, the wave packet starts to move from the field-free potential energy surface (PES) to the highest dressed PES and back again to the field-free PES. In the second half of the optical cycle, the wave packet executes the same type of motion as in the first half, but with the electric field pointing in the opposite direction. Then, the motion of the wave packet is repeated for the entire pulse duration. Therefore, the motion of wave packet along a potential energy surface in the optimal field is executed toward to coherently control product yields of chemical reactions.

Next, the optimal laser field and control of time-dependent occupation numbers which transfers the ground state to the higher excited states in order to produce the desired products of $\mathrm{CH}^{+}, \mathrm{C}^{+}$ and $\mathrm{C}^{++}$are obtained which the dissociation of methane is followed by the different channels

$$
\mathrm{CH}_{4} \stackrel{h v}{\longrightarrow} \begin{cases}\mathrm{CH}^{+}+3 \mathrm{H}+e & (a) \\ \mathrm{C}^{+}+4 \mathrm{H}+e & (\text { b }) \\ \mathrm{C}^{++}+4 \mathrm{H}+2 e & \end{cases}
$$

The results are shown in Fig. 4.

The system is driven selectively to $\mathrm{CH}^{+}, \mathrm{C}^{+}$and $\mathrm{C}^{++}$products, by maximum dissociation rates of $80 \%, 90 \%$, and $82 \%$, respectively. However, for higher excited states, the role of inner orbitals and coupling between them become more prominent. Therefore, it is necessary to consider more orbitals contributions, their symmetry and very large basis sets for calculations. As a 
consequence, by considering the effective orbitals in the dissociation, the predicted results of occupation numbers are improved in Fig. 4. The exact occupation numbers of these excited states have not been reached completely. This can be attributed to the fact that $100 \%$ overlap with the target state is not accessible due to ignoring some of very high lying levels during the pulse optimization.

\section{CONCLUSIONS}

In conclusion, in the present work, by combination of two recent QOCT and TDDFT methods, a valuable way is introduced for selectively desired channels in multi-atomic and multi-electron molecules dissociation. Based on the calculated exact energy diagrams for methane molecule by designing the theoretically tailored laser pulse, the dynamics of excited electrons, time evolution of the efficient occupation numbers and dissociation rates for valuable fragments are improved. Dissociation rates are extracted for interaction of $\mathrm{CH}_{4}$ molecule with Ti: Sapphire laser pulse with duration of 20fs (FWHM) and intensity in the range of $10^{14}-10^{15} \mathrm{Wcm}^{-2}$, using an iterative method. The optimally maximum dissociation rates for $\mathrm{CH}_{2}{ }^{+}, \mathrm{CH}^{+}, \mathrm{C}^{+}$and $\mathrm{C}^{++}$created from Coulomb explosion are obtained about $78 \%, 80 \%, 90 \%$, and $82 \%$, respectively. This shows remarkable improvement in comparison to the total dissociation rate of methane molecule and fragmental ion yields with a single laser pulse of a similar total energy which was only about $15 \%[19,29-31]$.

Acknowledgments: We acknowledge financial support from the Iranian National Science Foundation (INSF) and Sharif University of Technology's Office of Vice President for this research under Contract number No. 94027374. 


\section{References}

[1] X. Huang, D. Yang, L. Yao, Spectrochim. Acta Part A 130 ( 2014) 19-23.

[2] A. Saha, A. Kumar, P. D. Naik, Chem. Phys. Lett. 644 ( 2016) 73-78.

[3] E. Irani, R. Sadighi- Bonabi, A. Anvari, Chem. Phys. Lett. 604 (2014) 60-67.

[4] O.V. Boyarkin, S.I. Ionov, A.A. Kobakhidze, Spectrochim. Acta Part A 46(1990) 537-539.

[5] S. Palaniyappan, R. Mitchell, R. Sauer, I. Ghebregziabher, S.L. White, M. F. Decamp, and B.

C. Walker, Phys. Rev. Lett. 100 (2008) 183001 .

[6] A. Bellili, Y. Pan, M.M. Al Mogren, K.C. Lau, M. Hochlaf, Spectrochim. Acta Part A 164 ( 2016) 1-7.

[7] G.E. Murgida, D.A. Wisniacki, P.I. Tamborenea, F. Borondo, Chem. Phys. Lett. 496 (2010) $356-361$.

[8] J. Broeckhove, B. Feyen, P. Van Leuven, Journal of Molecular Structure: THEOCHEM, 261( 1992) 265-276.

[9] D. Tannor, and S.A. Rice, J. Chem. Phys. 83 (1985) 5013.

[10] A.H. Zewail, Femto Chemistry. Hackensack:World Scientific, 1994.

[11] R.S. Judson, and H. Rabitz, Phys.Rev. Lett. 68 (1992) 1500.

[12] X. Chu, and S. I. Chu, Phys. Rev. A 64 (2001) 021403 (R).

[13] J. Werschnik, and E.K.U. Gross, J. Phys. B 40 (2007) R175.

[14] J. Muth-Bohm, A. Becker, and F. H. M. Faisal, Phys. Rev. Lett. 85 (2000) 2280 .

[15] M. Smits, C. A. de Lange, A. Stolow, and D. M. Rayner, Phys. Rev. Lett. 93 (2004) 213003.

[16] Z. Zhao, T. Brabec, J. Phys. B: Atomic, Molecular and Optical Physics 39 (2006) L345.

[17] A. Castro, J. Werschnik, and E. K. U. Gross, Phys. Rev. Lett. 109 (2012) 153603.

[18] H.A. Navid, E.Irani, R. Sadighi-Bonabi, Spectrochim. Acta Part A 156 (2016) 118-122. 
[19] S. Zare, E. Irani, H. A. Navid, Z. Dehghani, A. Anvari, and R. Sadighi-Bonabi, Chem. Phys. Lett. 560 (2013) 60-65.

[20] S. Rasti, E. Irani, R. Sadighi-Bonabi, Journal of Molecular Structure 1083 (2015) 121-126.

[21] A. Castro, H. Appel, M. Oliveira, C. A. Rozzi, X. Andrade, F. Lorenzen, M. A. L. Marques, E. K. U. Gross, and A. Rubio, phys. stat. sol. (b) 243 (2006) 2465.

[22] X. Andrade, J. A. Rodriguez, D. A. Strubbe, M. J.T. Oliveira, F. Nogueira, A. Castro, J. Muguerza, A. Arruabarrena, S. G. Louie, A. A. Guzik, A. Rubio, M. A.L. Marques, Journal of Physics: Condensed Matter 24 (2012) 233202.

[23] M. A.L Marques, A. Castro, G. F. Bertsch, A. Rubio, Computer Physics Communications $151(2003) 60$.

[24] S. K. Son, Journal of Computational Physics 230 (2011) 2160-2173.

[25] P.R.T. Schipper, O. V. Gritsenko, S.J. A. van Gisbergen, and E.J. Baerends, J. Chem. Phys. 112 (2000) 1344.

[26] A. Castro, M. A. L. Marques, H. Appel, M. Oliveira, C. A. Rozzi, X. Andrade, F.Lorenzen, E. K. U. Gross, and A. Rubio, Phys. Status Solid B. 243 (2006) 2465 .

[27] W. Zhu, J. Botina, and H. Rabitz, J. Chem. Phys. 108 (1998) 1953 .

[28] M. J. Frisch, G.W. Trucks, et al. Gaussian03, Gaussian, Pittsburgh, Pa. RevisionA.1, 2003.

[29] M. Sharifi, F. Kong, S. L. Chin, H. Mineo, Y. Dyakov, A. M. Mebel, S. D. Chao, M. Hayashi, and S. H. Lin, J. Phys. Chem. A, 111 (38) (2007) 9405-9416.

[30] N. Ekanayake, B. L. Wen, L. E. Howard, S. J. Wells, M. Videtto, C. Mancuso,T. Stanev, Z. Condon, S. LeMar, A. D. Camilo, R. Toth, M. F. Decamp and B. C. Walker, J. Phys. B: At. Mol. Opt. Phys. 44 (2011) 045604. 
[31] S. Palaniyappan, R. Mitchell, R. Sauer, I. Ghebregziabher, S. L. White, M. F. Decamp, and B. C. Walker, Phys. Rev. Lett. 100 (2008) 183001. 


\section{Figure Captions:}

Fig. 1. Energy diagram of $\mathrm{CH}_{4}$ and its ionic dissociation products. The solid line is dissociation path for $\mathrm{CH}_{4}^{+}$, the dotted line, dash line and dash-dotted line are dissociation steps through different transition states for $\mathrm{CH}_{4}{ }^{++}$.

Fig. 2. (a) Initial laser pulse (green color) and the optimized laser pulse (blue color), (b) occupation analysis of relevant states as a function of time, during the process.

Fig. 3. Snapshots of the four lowest eigenstates distributions in the plane XY for (a) $1^{\text {st }}$, (b) $2^{\text {nd }}$, (c) $3^{\text {rd }}$, (d) $4^{\text {th }}$ state.

Fig. 4. The time evolution of the efficient occupation numbers for (a) first dissociation channel, (b) second dissociation channel, (c) third dissociation channel. 
Figures:

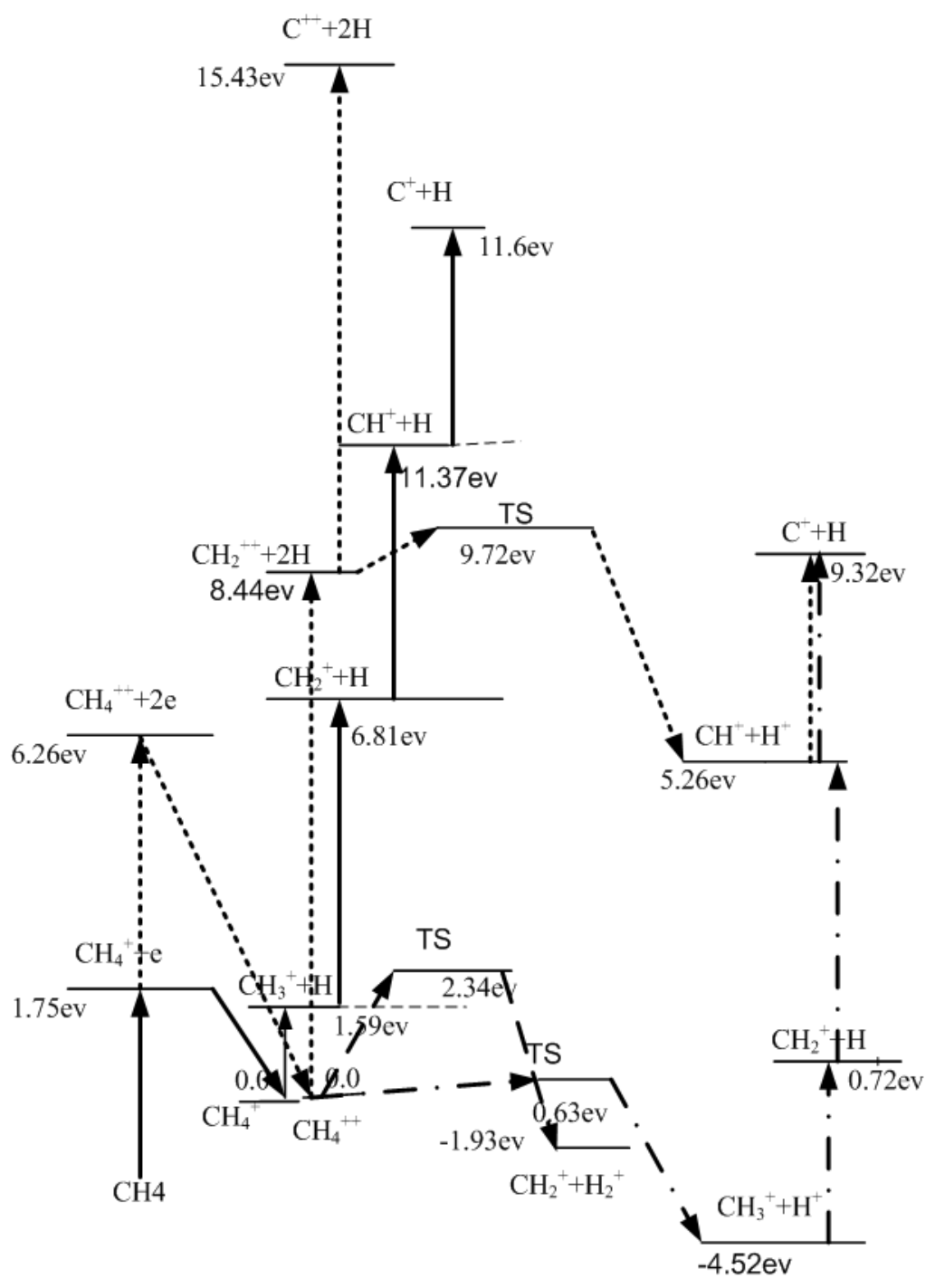

Fig. 1 


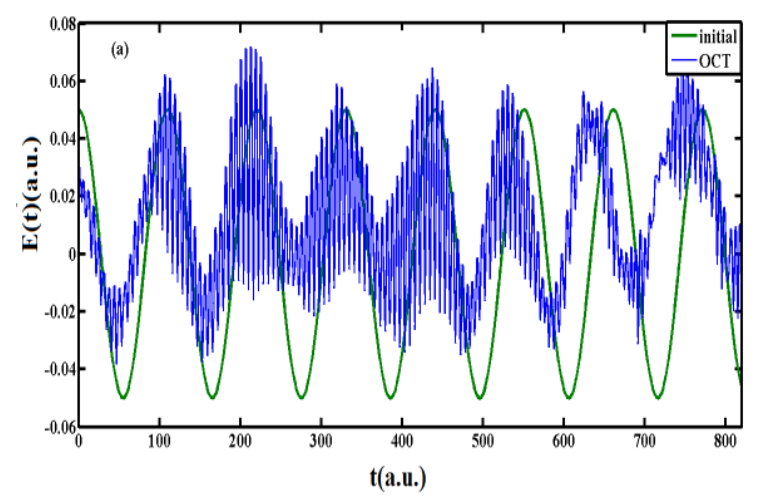

Fig. 2a

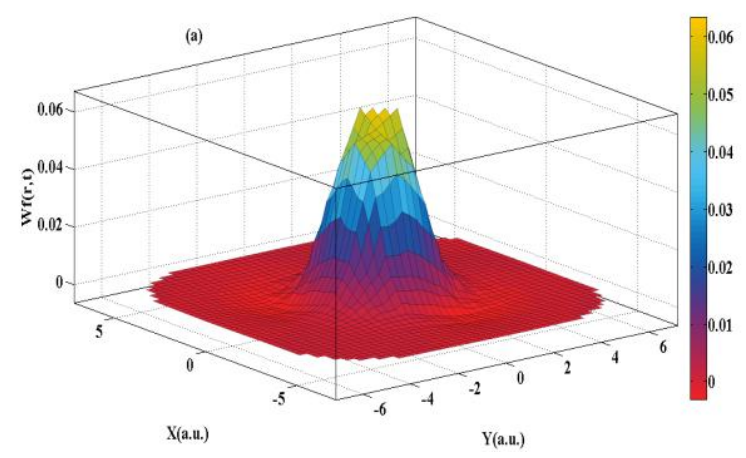

Fig. 3a

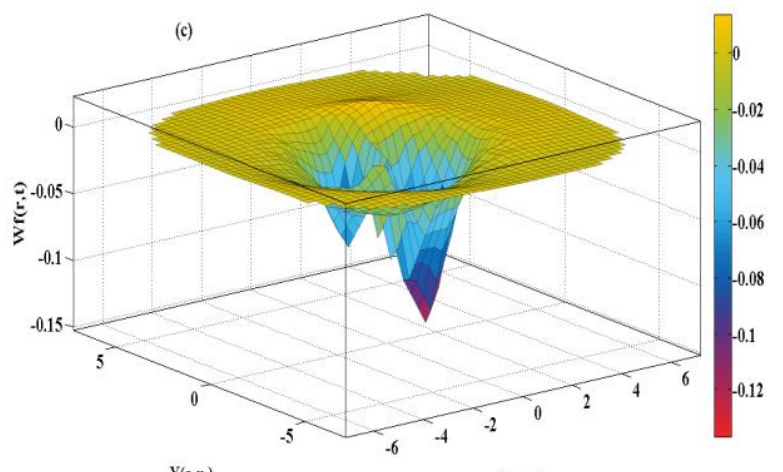

X(a.u.)

Fig. 3c

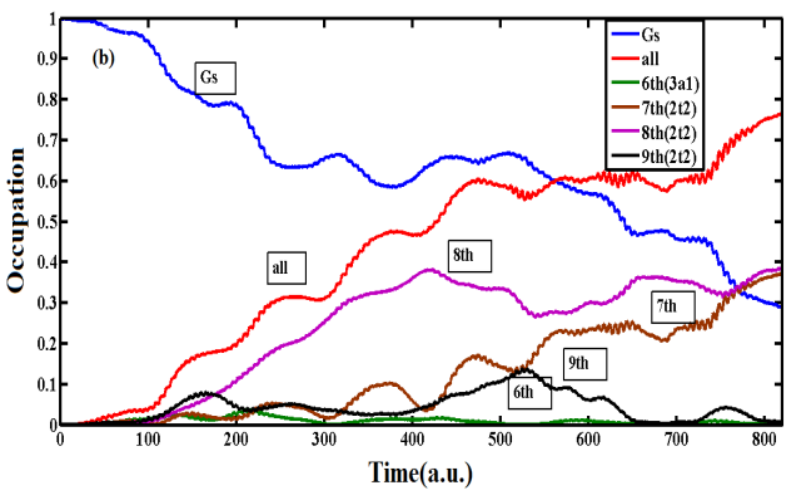

Fig. 2b

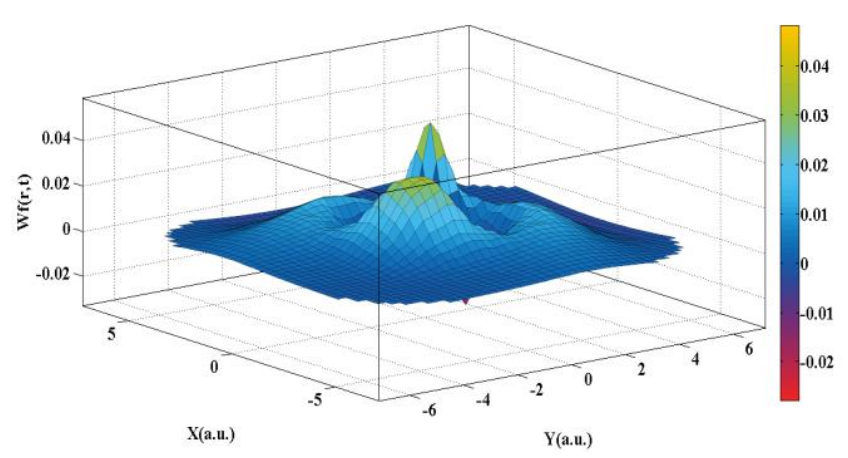

Fig. 3b

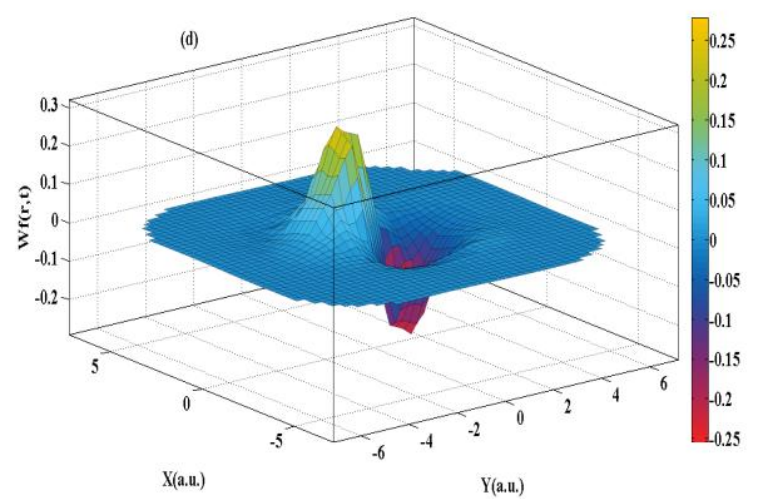

Fig. 3d 


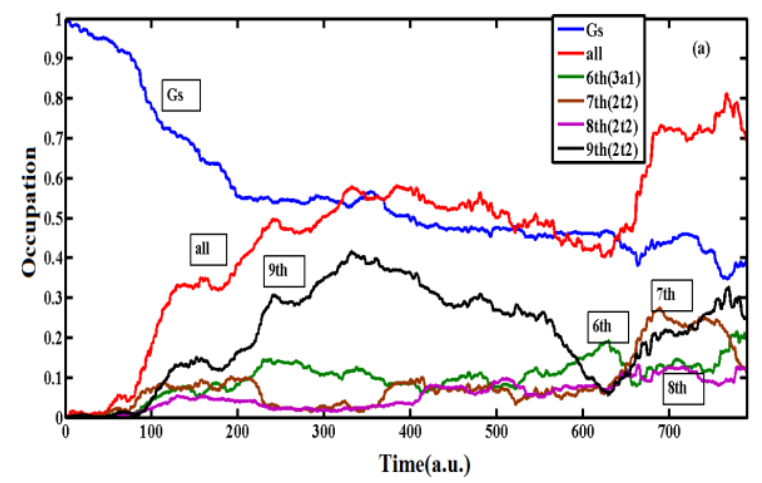

Fig. 4a

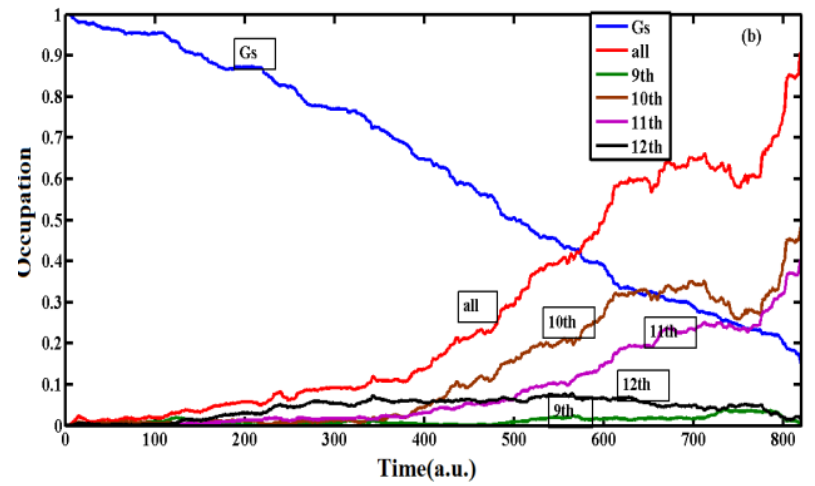

Fig. $4 b$

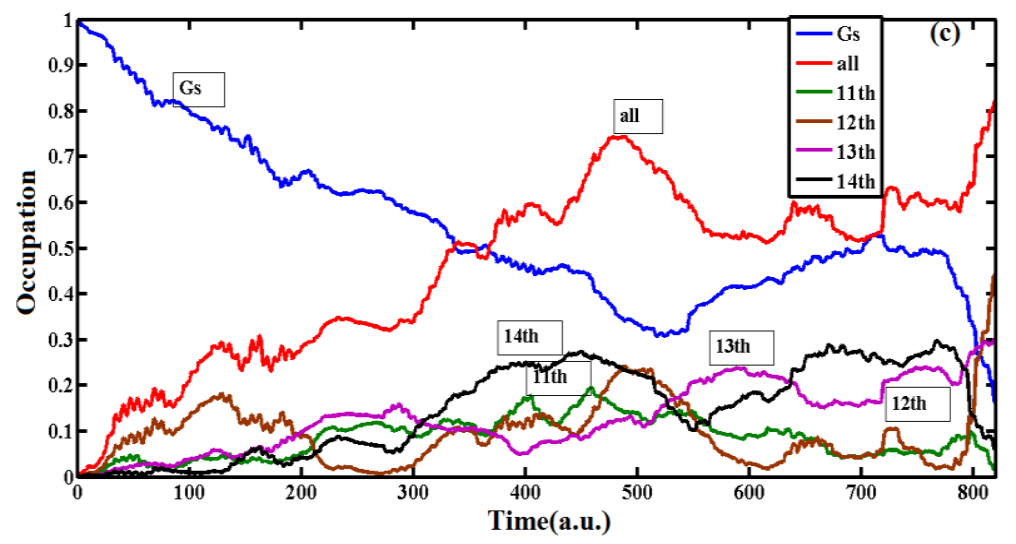

Fig. 4c 


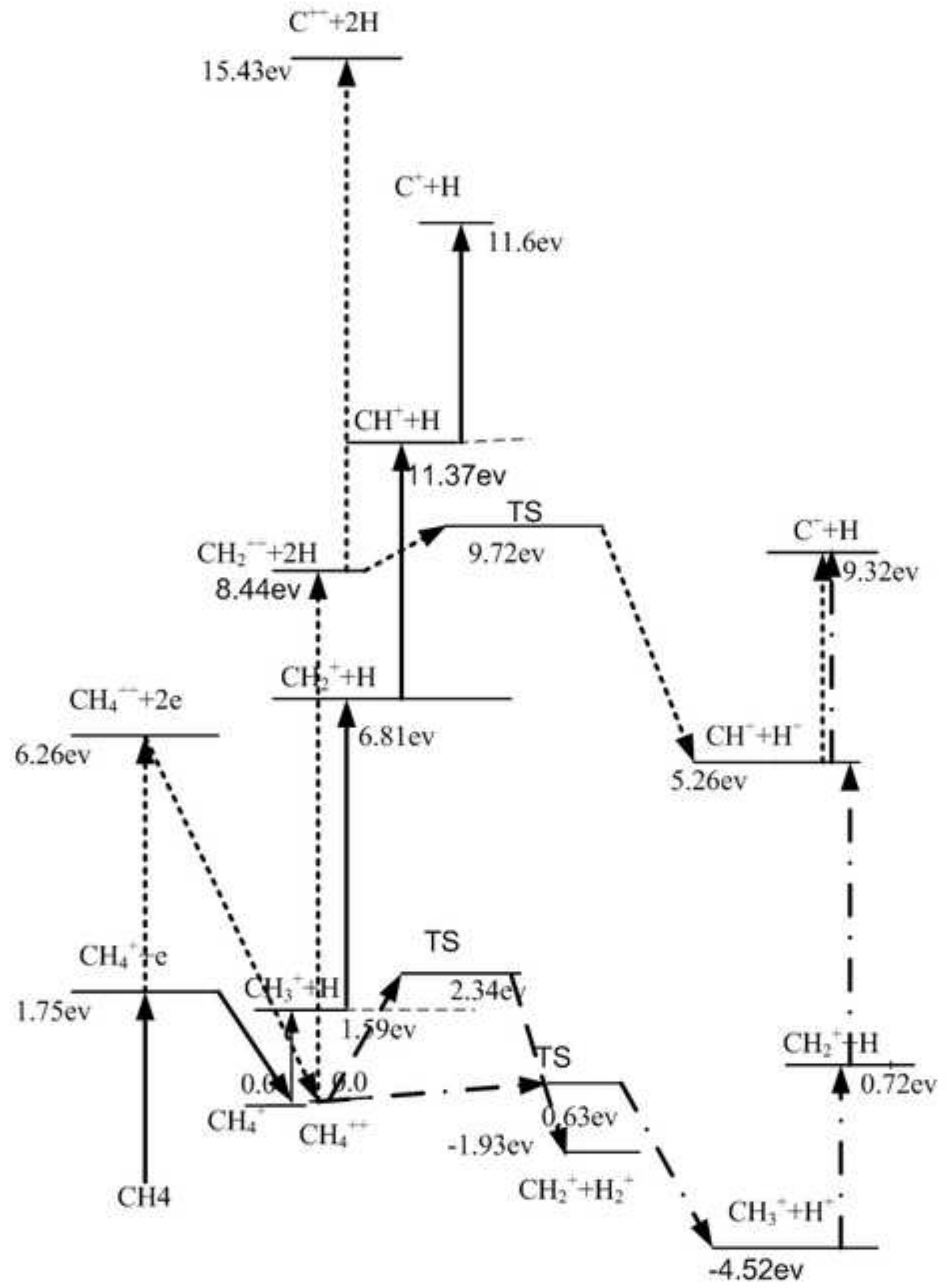




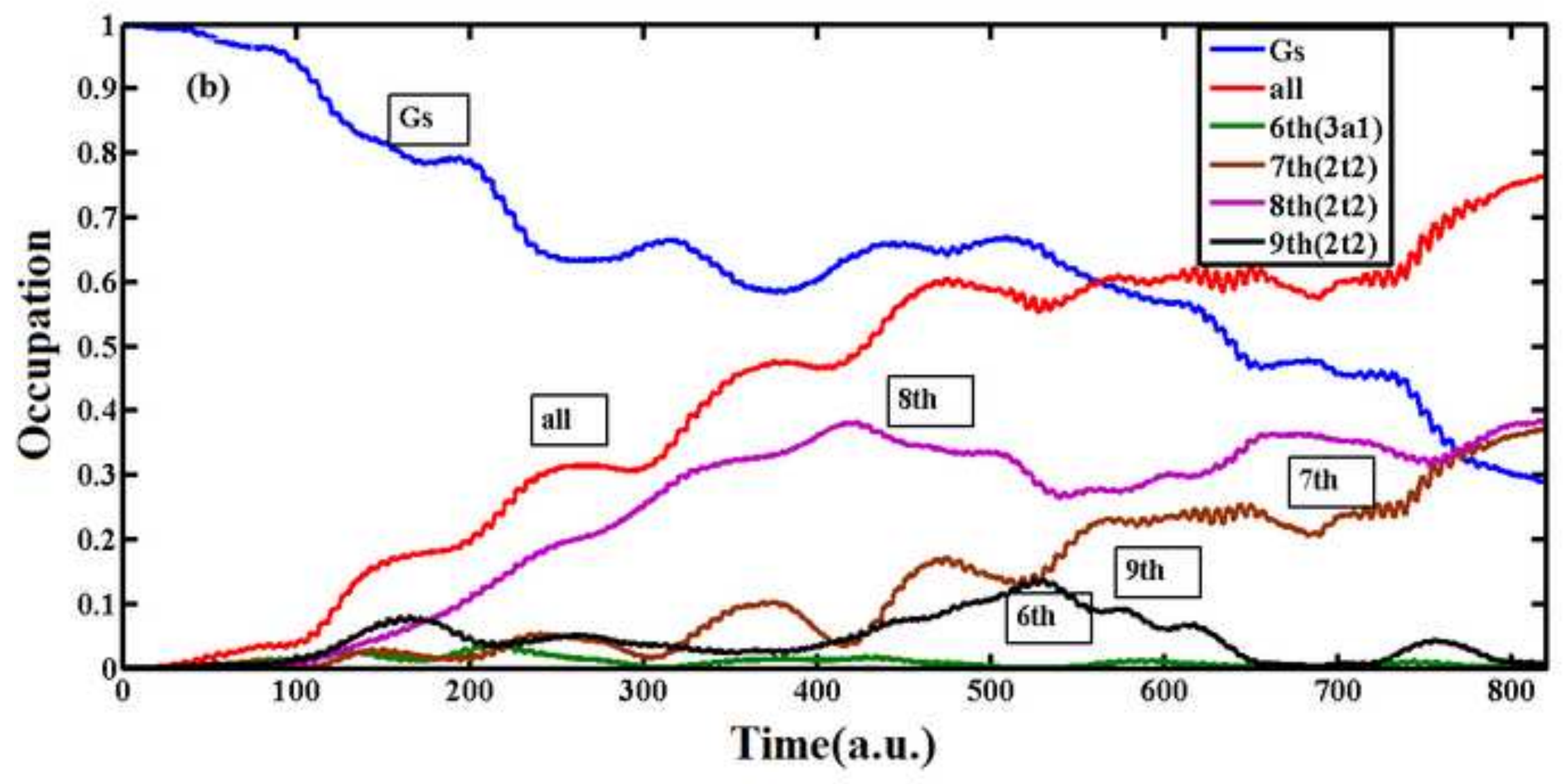

